\title{
Pharyngeal carriage of Neisseria meningitidis before and after treatment of meningococcal disease
}

\author{
NINA WEIS and INGA LIND
}

Statens Seruminstitut, Neisseria Department, Artillerivej 5, DK-2300 Copenhagen S, Denmark

\begin{abstract}
Summary. The aim of the study was to determine whether patients with meningococcal disease carry meningococci in the throat both before and after treatment for the disease. During the 7 months of the study 106 patients with confirmed meningococcal disease were admitted to Danish hospitals, of whom $77(73 \%)$ had a throat swab examined at least once and were included in the study. Sixty-two patients were examined on admission and 52 were examined on discharge; 37 were examined on both occasions. On admission, meningococci were isolated from $18(49 \%)$ of 37 throat specimens examined selectively for pathogenic Neisseria spp. Meningococci were not isolated from any throat specimen taken on discharge from hospital; 47 (90\%) of 52 of these specimens had been examined adequately. From an observed carriage rate of 0 out of 47 it can be judged that the carrier rate does not exceed $6.4 \%$ ( $95 \%$ confidence limit). From these results we conclude that it is unlikely that patients who have been treated for meningococcal disease according to the regimens used in Denmark can be the source of infection for secondary cases.
\end{abstract}

\section{Introduction}

Meningococcal disease develops after the transmission of droplets that contain a virulent strain of Neisseria meningitidis to a susceptible person, usually from an asymptomatic carrier. ${ }^{1,2}$ Although patients with meningococcal disease are seldom the source of the infection, ${ }^{1}$ meningococcal disease occurs 1000 times more frequently in household contacts of such patients than in the general population during a nonepidemic period. ${ }^{3}$

Pharyngeal carriage of meningococci is common in healthy individuals. The carriage rate has been found to vary from a small percentage among infants to $25-35 \%$ among teenagers and young adults ${ }^{4,5}$ and thereafter to decline with increasing age. ${ }^{4,6}$ Carriage rates of $40-60 \%$ have been found consistently among young adults who live in closed communities, such as military recruits or miners. ${ }^{68}$ Virulent meningococci were isolated from only $1.4-1.6 \%$ of healthy individuals. ${ }^{4,9}$ During epidemics the percentage of carriers of virulent strains can rise to $17 \%$ in family contacts ${ }^{10}$ and to an overall average of $35 \%$ (range $1-74 \%$ ) in Army Recruit Centres. ${ }^{11}$

Measures to prevent secondary cases of meningococcal disease comprise careful observation of close contacts of patients with meningococcal disease for the development of symptoms, and chemoprophylaxis or vaccination, or both.

The recommendation in the United Kingdom is that not only household contacts of patients with meningo-

Received 7 March 1994; revised version accepted 19 May 1994. coccal disease but also patients discharged from hospital after treatment for meningococcal disease should receive chemoprophylaxis. ${ }^{12}$ This decision is based on the assumption that treatment with penicillin does not eradicate meningococci from the pharynx in every case. ${ }^{13}$

Because we are aware of only one other report on the frequency of carriage in patients discharged from hospital after treatment for meningococcal disease, ${ }^{10}$ we have investigated the frequency with which Danish patients harboured meningococci in the throat before and after treatment.

As the results of this study showed a striking geographical difference in the percentage of patients from whom meningococci were isolated from the throat on admission to hospital, a retrospective study of the media used for the primary isolation of meningococci from throat swabs was included.

\section{Materials and methods}

Between 1 Aug. 1988 and 1 March 1989106 patients with confirmed meningococcal disease were admitted to Danish hospitals. Meningococci were isolated from cerebrospinal fluid or blood cultures or petechiae, or any combination, from 98 of these and from either tracheal secretion (one) or from throat swabs (seven) of a further eight patients with a clinical diagnosis of meningococcal disease. The table shows some characteristics of isolates from these patients. At least one throat swab was examined from $77(73 \%)$ of the 106 patients, and these were included in the study. 
Table. Origin and results of serogroup determination of meningococcal isolates from 106 patients with meningococcal disease in Denmark during the period 1 Aug. 1988 to 1 March 1989

\begin{tabular}{lcccc} 
& \multicolumn{4}{c}{ Number of strains } \\
\cline { 2 - 5 } Source of isolate* & Total & $\begin{array}{c}\text { Serogroup } \\
\text { B }\end{array}$ & $\begin{array}{c}\text { Serogroup } \\
\text { C }\end{array}$ & $\begin{array}{c}\text { Other } \\
\text { serogroups }\end{array}$ \\
\hline Spinal fluid & 57 & 45 & 10 & $2(\mathrm{Y}, \mathrm{Z})$ \\
Blood & 25 & 16 & 8 & $1(\mathrm{X})$ \\
Spinal fluid and blood & 13 & 10 & 3 & 0 \\
Petechiae & 1 & 1 & 0 & 0 \\
Petechiae and throat & 1 & 1 & 0 & 0 \\
Biopsy & 1 & 1 & 0 & 0 \\
Tracheal secretion & 1 & 1 & 0 & 0 \\
Throat & 7 & 5 & 2 & 0 \\
Total & 106 & 80 & 23 & 3 \\
\hline
\end{tabular}

*Only one isolate from each patient is included.

Isolates from patients with meningococcal disease are sent to the Neisseria Department, Statens Seruminstitut, Copenhagen, for the determination of serogroup, serotype, sero-subtype and in some cases other epidemiological markers such as resistance to sulphonamide. Whenever an isolate was received, the nursing staff at the hospital were requested to take a throat swab from the convalescent patient. The patients usually remained in hospital for 2 days after the cessation of treatment with antibiotics. Information as to whether throat swabs had been taken from patients on admission to hospital and whether meningococci had been isolated at the local clinical microbiology laboratory was obtained retrospectively.

The two media routinely used for the bacteriological examination of throat swabs were horse blood $5 \%$ agar and Danish GC chocolate agar made selective for pathogenic neisseriae by the addition of lincomycin $1 \mu \mathrm{g} / \mathrm{ml}$, polymyxin B sulphate $25 \mathrm{U} / \mathrm{ml}$, mycostatin $20 \mathrm{U} / \mathrm{ml}$ and trimethoprim lactate $3 \mu \mathrm{g} / \mathrm{ml}$.

\section{Results}

\section{Pharyngeal carriage of meningococci on admission to hospital}

Throat swabs were taken from $62(81 \%)$ of the 77 patients included in the study on admission to hospital. Of these patients. $22(35 \%)$ carried meningococci. In two of the 40 patients from whom no meningococci were isolated, antibiotic treatment had been started before the throat swabs were taken.

A carriage rate of $35 \%$ in patients with meningococcal disease was considered to be surprisingly low ${ }^{14.15}$ and details of the culture media used for processing the throat swabs in local clinical microbiology laboratories were obtained for $57(92 \%)$ of the 62 cases. Of 37 swabs which had been inoculated on selective Danish GC chocolate agar medium, meningococcal isolates were isolated from $18(49 \%)$. Of another 20 throat swabs inoculated only on to horse blood $5 \%$ agar, meningococci were isolated from only one $(5 \%)$. The difference between the number of isolates found on horse blood $5 \%$ agar and the number isolated on selective Danish GC chocolate agar medium is statistically significant (Fisher's exact test, $\mathrm{p}=0.001$ ).

\section{Pharyngeal carriage of meningococci on discharge from hospital}

From 37 of the 62 patients from whom throat swabs were taken on admission to hospital, throat swabs were also taken on discharge. No meningococci were isolated from any of these 37 patients after the cessation of treatment with antibiotics. Ten had been pharyngeal carriers of meningococci on admission to hospital. Of 25 patients from whom throat swabs were taken on admission but not on discharge from hospital, $12(48 \%)$ had been meningococcal carriers. Of fifteen $(34 \%)$ of 44 patients from whom no throat swab was taken on admission, a throat swab was taken on discharge, and no meningococci were isolated from any of these. Forty-seven $(90 \%)$ of the 52 throat swabs taken on discharge from hospital had been inoculated on selective Danish GC chocolate agar medium. From an observed carriage rate of zero out of 47 , it can be deduced with $95 \%$ confidence that the carriage rate does not exceed $6.4 \%$.

\section{Discussion}

In Denmark, as in other countries such as the UK and the USA, there is a national surveillance system for meningococcal disease that includes circulars describing measures for the prevention of secondary cases. ${ }^{3.12 .16}$ The main precaution to reduce the risk of a serious course of meningococcal disease in close contacts is careful observation for symptoms. The decision as to whether to offer prophylactic antibiotic treatment or vaccination, or both, to close contacts has to be considered in connection with each individual case of meningococcal disease. In 1988-1989 the prophylaxis for adults was rifampicin $600 \mathrm{mg}$ orally 
twice a day for 2 days. Since 1992, the recommended regimen has been ciprofloxacin $500 \mathrm{mg}$ orally as a single dose. ${ }^{16}$ The purpose of prophylactic antibiotics is to eliminate carriage of the virulent strains before they cause invasive disease or are spread to potentially susceptible individuals. As there is an increased risk of meningitis for members of the household of a patient, ${ }^{1.3}$ "close contacts" are usually defined as persons belonging to the patient's household.

In Britain, prophylactic antibiotics are also given to convalescent patients before discharge from hospital. ${ }^{12}$ The work of Abramson and Spika ${ }^{13}$ was part of the basis for this decision. Their study included 14 patients with meningococcal disease; $10(71 \%)$ of the patients were carriers on admission to hospital, none was a carrier after 1 day of therapy, one patient $(7 \%)$ was a carrier when discharged, and four patients $(29 \%)$ were carriers a week later. ${ }^{13}$ The authors were uncertain whether the persistence of $N$. meningitidis in the upper respiratory tract is due to failure of intravenous antibiotics to eradicate the organism or to recolonisation from a close contact. In an earlier study, Blakebrough and Gilles ${ }^{10}$ found that none of 44 patients with serogroup A meningococcal disease was carrying $N$. meningitidis after treatment for $24 \mathrm{~h}$ and that only one became a carrier of serogroup A meningococci again within 6 weeks. This patient returned to his family, four out of five of whom were carriers of serogroup A meningococci throughout.

In a later study by Alvez et al. ${ }^{17}$ throat swabs were taken on the day of discharge (designated day 0 ) and 10 days later from 48 children in whom meningococcal disease was confirmed by culture of $N$. meningitidis from cerebrospinal fluid or blood, or both. On day 0 , alternate patients were given placebo or prophylaxis with rifampicin. On day 0 , meningococci were detected in throat cultures from three $(6.25 \%)$ of the 48 children. On day 10, throat cultures of four children $(8.34 \%)$ yielded meningococci. Rifampicin had been administered to all of the three children with positive day 0 throat cultures, one of whom remained positive

\section{References}

1. Broome CV. The carrier state: Neisseria meningitidis. J Antimicrob Chemother 1986; 18 Suppl A: 25-34.

2. Schwartz B, Moore PS, Broome CV. Global epidemiology of meningococcal disease. Clin Microbiol Rev 1989; 2: S118-S124.

3. The Meningococcal Disease Surveillance Group. Meningococcal disease. Secondary attack rate and chemoprophylaxis in the United States, 1974. JAMA 1976; 235 : 261-265.

4. Cartwright KAV, Stuart JM, Jones DM, Noah ND. The Stonehouse survey: nasopharyngeal carriage of meningococci and Neisseria lactamica. Epidemiol Infect 1987; 99: $591-601$.

5. Olsen SF, Djurhuus B, Rasmussen $\mathrm{K}$ et al. Pharyngeal carriage of Neisseria meningitidis and Neisseria lactamica in households with infants within areas with high and low incidences of meningococcal disease. Epidemiol Infect 1991; 106: 445-457.

6. Holten E, Bratlid D, Bøvre K. Carriage of Neisseria meningitidis in a semi-isolated arctic community. Scand $J$ Infect Dis $1978 ; 10: 36-40$. on day 10, suggesting either a lack of response to rifampicin or re-infection. Three of the four children who were carriers on day 10 had had negative throat culture on day 0 and had not received prophylactic rifampicin treatment, suggesting re-infection from the family or community. Alvez et al. did not recommend prophylactic treatment to patients discharged from hospital after treatment for meningococcal disease. Cartwright et al..$^{18}$ found that antibiotics used in the treatment of meningococcal disease successfully eliminated nasopharyngeal carriage of meningococci in 13 of 14 cases in whom the nasopharynx was swabbed 3-39 days after the completion of treatment. These results suggest that the parenteral antibiotics used can be effective in eliminating the nasopharyngeal carriage of meningococci.

In the present study, the carriage rate among patients adequately examined when admitted to hospital was $49 \%$. This finding was in agreement with those of Sippel and Girgis ${ }^{14}$ and Cartwright and Jones. ${ }^{15}$ Throat swabs were taken from $52(68 \%)$ of 77 patients on discharge from hospital. None of these patients, including 10 of the patients from whom meningococci were isolated from the throat before treatment, were carriers when discharged. As the patients were not examined later, the presence of meningococci that were temporarily inhibited by the antibiotics given as treatment cannot be excluded.

In summary, these studies showed that the percentage of patients who are pharyngeal carriers after treatment for $24 \mathrm{~h}$ is zero, that it is low after completion of treatment or on discharge from hospital, and that the rate afterwards is likely to increase with time. On the other hand, more studies with serotyping and genotyping are needed to ascertain whether a strain of $N$. meningitidis isolated from a patient recovering from meningococcal disease is identical to the original pathogenic strain.

We thank all the hospital staff who made this study possible and M. Weis Bentzon, Department of Biostatistics, Statens Seruminstitut, for his assistance with statistical analysis.

7. Reller LB, MacGregor RR, Beaty HN. Bactericidal antibody after colonization with Neisseria meningitidis. $J$ Infect Dis 1973; 127: 56-62.

8. Renkonen O-V, Sivonen A, Visakorpi R. Effects of ciprofloxacin on carrier rate of Neisseria meningitidis in army recruits in Finland. Antimicrob Agents Chemother 1987; 31 : $962-963$.

9. Saez-Nieto JA, Dominguez JR, Monton JL et al. Carriage of Neisseria meningitidis and Neisseria lactamica in a school population during an epidemic period in Spain. $J H y g$ 1985; 94: 279-288.

10. Blakebrough IS, Gilles HM. The effect of rifampicin on meningococcal carriage in family contacts in northern Nigeria. J Infect 1980; 2 : 137-143.

11. Edwards EA, Devine LF, Sengbusch CH, Ward HW. Immunological investigations of meningococcal disease. III. Brevity of group C acquisition prior to disease occurrence. Scand $J$ Infect Dis 1977; 9: 105-110.

12. Jones DM. Control of meningococcal disease. Chemoprophylaxis for carriers and some contact groups. BMJ 1989; 298: 542-543.

13. Abramson JS, Spika JS. Persistence of Neisseria meningitidis in the upper respiratory tract after intravenous antibiotic 
therapy for systemic meningococcal disease. $J$ Infect Dis $1985 ; 151: 370-371$

14. Sippel JE, Girgis NI. Throat culture from patients with meningococcal meningitis. J Clin Pathol 1990; 43:610-611.

15. Cartwright KAV. Jones DM. Value of throat swabs from index cases of meningococcal meningitis. J Clin Pathol 1990: 43 : 438 .

16. Sundhedsstyrelsen: Cirkulære og vejledning om profylaktiske foranstaltninger ved meningokoksygdom. Sundhedsstyrelsens cirkulære af 6. oktober 1992, Copenhagen, 1992.

17. Alvez F, Aguilera A, Garcia-Zabarte A, Castro-Gago M. Effect of chemoprophylaxis on the meningococcal carrier state after systemic infection. Pediatr Infect Dis J 1991; 10: 700.

18. Cartwright KAV, Stuart JM, Robinson PM. Meningococcal carriage in close contacts of cases. Epidemiol Infect 1991; 106: 133-141. 